\title{
Comparison of the Production of Nanostructures on Bulk Metal Samples by Picosecond Laser Ablation at Two Wavelengths for the Fabrication of Low-reflective Surfaces
}

\author{
Tamás CSIZMADIA ${ }^{* 1}$, Tomi SMAUSZ ${ }^{* 2}$, Csaba TÁPAI $^{* 1}$, Judit KOPNICZKY $^{* 1}$, Xi WANG $^{* 3}$, Martin EHRHARDT $^{* 3}$, \\ Pierre LORENZ ${ }^{* 3}$, Klaus ZIMMER ${ }^{* 3}$, László OROSZ ${ }^{* 1}$, Erika VARGA ${ }^{* 4}$, Albert OSZKÓ ${ }^{* 4}$, Béla HOPP ${ }^{* 1}$ \\ ${ }^{* 1}$ Department of Optics and Quantum Electronics, University of Szeged, H-6720 Szeged, Dóm tér 9, Hungary \\ ${ }^{* 2}$ MTA-SZTE Research Group on Photoacoustic Spectroscopy, University of Szeged, 6720 Szeged, Dóm tér 9, Hungary \\ ${ }^{*}$ Leibniz-Institut für Oberflächenmodifizierung e. V., Permoserstr. 15, 04318 Leipzig, Germany \\ ${ }^{* 4}$ Department of Physical Chemistry and Materials Science, University of Szeged, Aradi vértanúk tere 1, Szeged H-6720,
} Hungary

\begin{abstract}
Nanostructure formation on bulk metals (copper, gold, and silver) by picosecond ( $F W H M=10 \mathrm{ps)} \mathrm{Nd:YAG} \mathrm{laser}$ irradiation was studied aiming at the production of low-reflectivity surfaces. The experiments were performed at two distinct wavelengths $(\lambda=355$ and $1064 \mathrm{~nm})$ using $20 \mathrm{kHz}$ repetition frequency. The fluence was varied in the $1-11 \mathrm{~J} / \mathrm{cm}^{2}$ range, while the samples were shot by an average pulse number from 0 to 50 depending on the scanning speed of the applied galvanometric scanner. The reflectivity of the treated surfaces was recorded with a visible near-infrared microspectrometer in the 450-800 nm range. Morphological investigations of the irradiated metal surfaces were performed with scanning electron microscope, which showed that mainly two types of nanostructures can be responsible for the reflectivity decrease depending on the type of the illuminated metal. Finite element modeling was performed to simulate the laser absorption-induced heating of the illuminated samples, which helps in the understanding of the structure formation process. It was found that two main processes take place in the production of micro- and nanostructures on the surface; the ejection and falling back of molten metal droplets, and the back scattering and aggregation of nanoparticles.
\end{abstract}

DOI: 10.2961/jlmn.2015.02.0001

Keywords: black metal, darkening, picosecond, copper, gold, silver, reflectivity, pulse laser

\section{Introduction}

In recent years, several pulsed laser processing technologies have been demonstrated for producing random nano- and microstructures on the surface of numerous metals [1-3]. One important feature of this laser irradiation-induced surface patterning is that it can be used for modifying optical properties of the treated area [4-6]. Spectroscopic studies showed that due to the nanotexturing of the sample surface, originally highly reflective materials can turn into almost completely absorptive in a broad spectral range (from near UV to near IR), when the proper experimental conditions are applied.

This darkening effect was mainly reported in the femtosecond region [7-10]; however, there were also some early trials in the longer pulse regions. Fan et al. utilized a picosecond laser beam for modifying the reflectivity property of bulk copper. This research was focused on varying the interval between the adjacent lines of the scanned area, while the fluence and the scanning speed were kept constant [11]. In another experiment Y. C. Guan et al. observed reflectivity decrease on the surfaces of magnesium alloys after nanosecond pulse laser irradiations. These experiments were carried out in the presence of atmospheric air environment and the darkening effect was not only attributed to the light trapping properties of the rough surface, but also to oxidation and other compositional variations of the illuminated surfaces $[12,13]$. Therefore metal darkening with longer pulses is still in its early stage and has open questions concerning both the reflectivity decrease mechanism and the structure forming process on the surface. In addition to the random micro- and nanostructures, absorptivity increase was also reached via laser-induced periodic surface structures with a microscale period formed over the above-mentioned random surface structures [14-16].

In the past few years, the interest has grown to find the explanations of the change in the absorptive properties of the irradiated spots and to search for the potential applications. There is large interest in light trapping and manipulation techniques including antireflection coatings, surface texturing, and optical absorption enhancement to improve power conversion efficiencies in photovoltaic devices generally [17-19]. The nanostructured, darkened metal surfaces also have important applications in other broad areas of research and developments, such as plasmonics, optoelectronics, metal colorization, absorbers of solar light, broadband thermal sources etc.

Although several papers have been presented so far that aimed to describe the optical property modifications in correlation with the changes of the surface morphology of metals in case of femtosecond laser pulses [20-22], the method was not detailed in the picosecond region yet, which can help answer open questions concerning both the evolution process of the relevant micro- and nanostructures and the mechanism of 
the reflectivity decreasing. In the following study, different metal (gold, silver, copper) targets were irradiated by picosecond Nd:YAG laser pulses to form optimal surface patterns resulting in low reflectance and the development of surface structures at different processing parameters including two distinct processing wavelengths was investigated.

\section{Experimental}

High purity bulk metal (copper, silver, and gold) plates (from Goodfellow) were irradiated by pulses of a $\mathrm{Nd}$ :YAG laser $(F W H M=10 \mathrm{ps})$ operated at basic IR and third harmonic UV wavelengths $(1064 \mathrm{~nm}$ and $355 \mathrm{~nm}$, respectively) at $20 \mathrm{kHz}$ repetition rate. The laser beam that was focused onto the sample surface into a circular spot with $30 \mu \mathrm{m}$ diameter was scanned by galvanometric scanner across a $1 \mathrm{~mm} \times 1 \mathrm{~mm}$ sized area of the targets. By varying the scanning speed, the pulse number reaching a given area could be varied from 0 to 50 on average. The laser fluence was altered between 0.94 and $11.1 \mathrm{~J} / \mathrm{cm}^{2}$.

\section{Results}

\subsection{Reflectivity measurements}

All the sample irradiations were performed at atmospheric pressure and at room temperature. After the metal plates were illuminated, the reflectivity change of the treated surfaces was visible by naked eye for all the applied parameter values (Figure 1).

The reflectivity of the processed areas were measured by a visible near-infrared microspectrometer equipped with an $\mathrm{NA}=0.12$ objective, with which the light backscattered from the investigated circular area having approximately $100 \mu \mathrm{m}$ diameter was detected in the wavelength range of $450-800 \mathrm{~nm}$. The recorded spectra were normalized to the reflectivity of the untreated, non-polished metal samples.

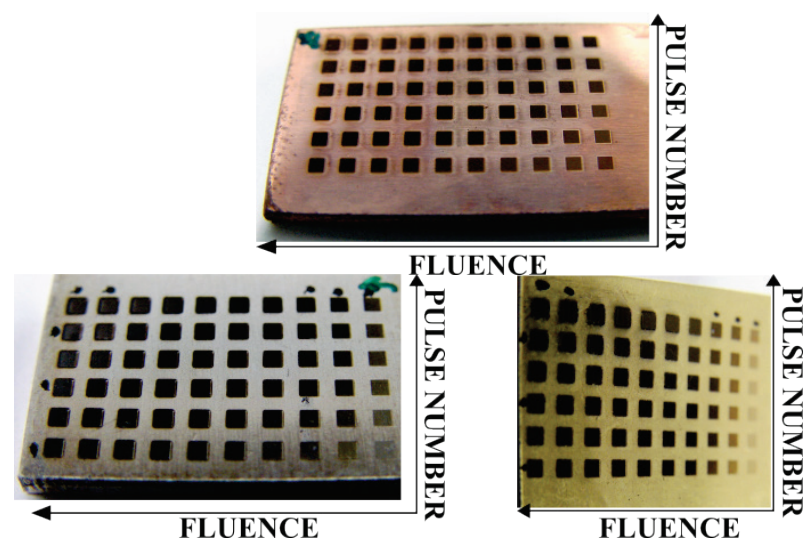

Fig. 1: Photographs of the laser-treated spot matrices on copper, silver, and gold plates, respectively.

It is important to note that due to the relatively low numerical aperture of the microspectometer's objective, mainly the intensity of the specular reflected light was measured and some of the diffusely reflected light is neglected. Figure 2 shows the recorded normalized reflectivity spectra of gold targets as a function of the laser fluences at the two processing wavelengths. The applied average pulse number was 50 . It was found that the reflectivity of the processed surfaces already significantly decreased at the lowest applied fluence in case of all the three investigated metals. Moreover, the similar tendencies of the curves showing roughly constant reflectivity reduction, which indicates that the light trapping phenomena does not significantly depend on the wavelength of the reflected light in the investigated spectral range.
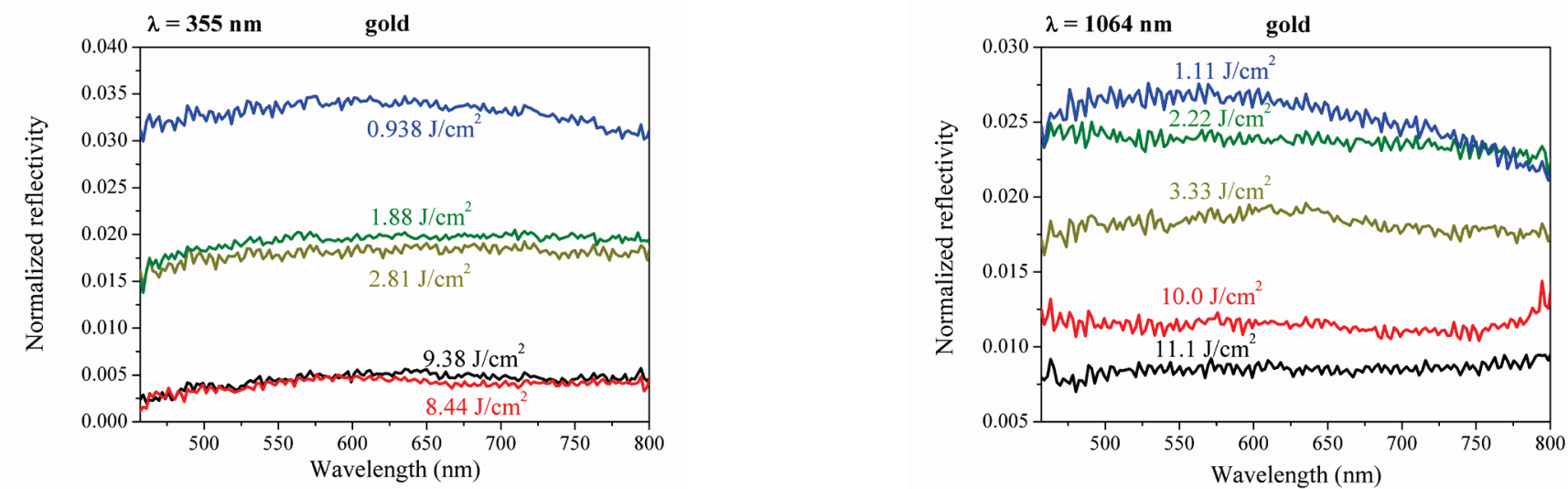

Fig. 2: Normalized reflectivity spectra of gold targets irradiated with $50 \mathrm{Nd}$ :YAG laser pulses at different applied fluences. 

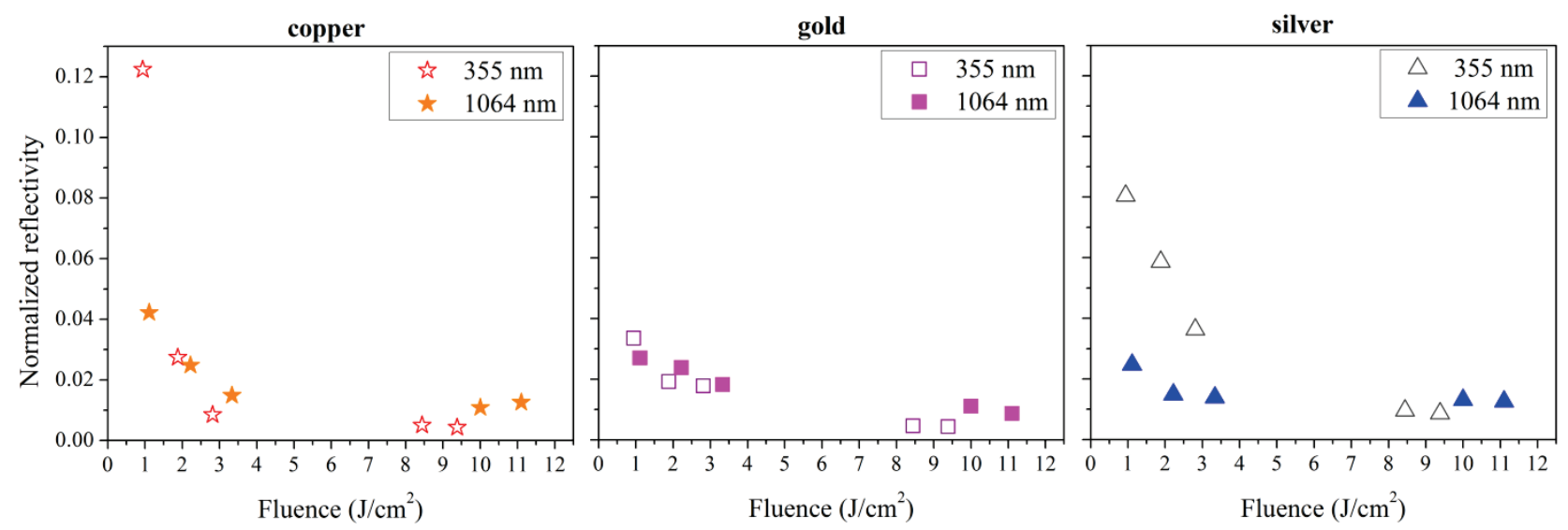

Fig. 3: Relative reflectivity measured at $550 \mathrm{~nm}$ for the three investigated metals as a function of the applied laser fluence. The samples were irradiated with 50 laser pulses at two operational wavelengths.

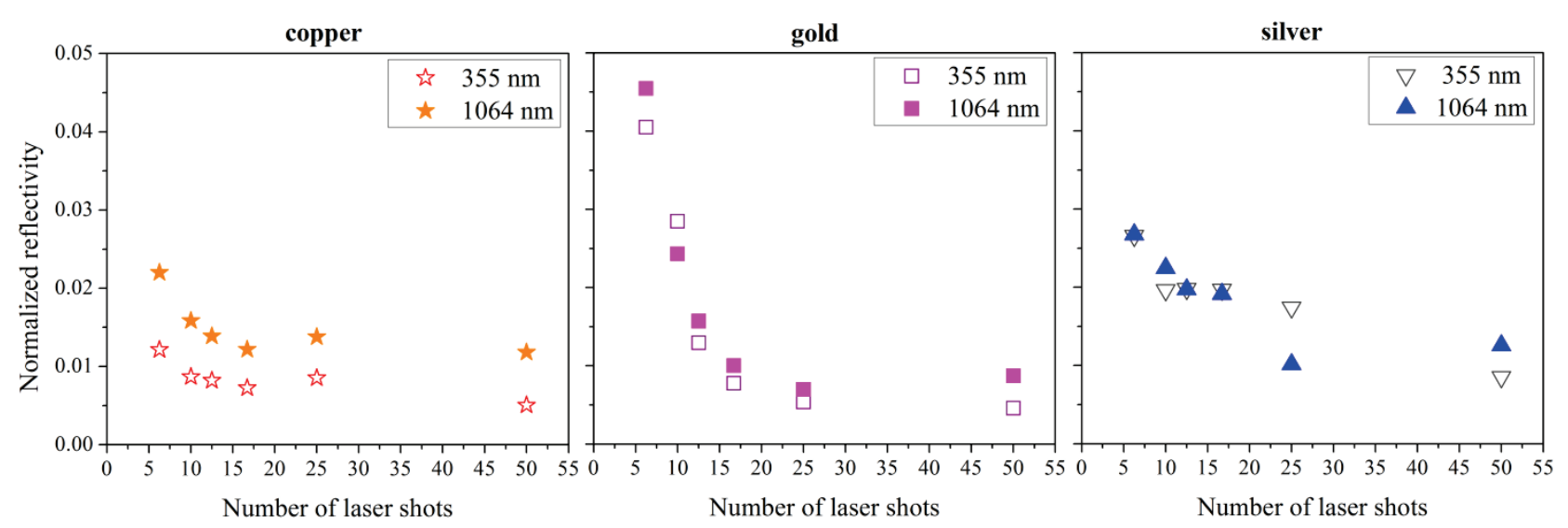

Fig. 4: The dependence of the darkening effect on the applied laser pulse number. The samples were irradiated at two applied wavelengths with maximum fluence (about $10 \mathrm{~J} / \mathrm{cm}^{2}$ depending on the applied wavelength).

Figure 3 shows the dependence of the normalized reflectivities at $\lambda=550 \mathrm{~nm}$ on the laser fluence using 50 pulses. It was found that the normalized reflectivity drastically decreased with increasing fluence in the range of $0-3 \mathrm{~J} / \mathrm{cm}^{2}$ and reduced below 0.03 in all cases at $3 \mathrm{~J} / \mathrm{cm}^{2}$. Above this value the normalized reflectivities remains quasi constant in case of silver and copper, while further slow decrease occurs in the reflectivity of gold. Regarding the two processing wavelengths, in case of silver a slight difference can be seen between the tendencies of the normalized reflectivity-fluence dependency in the low fluence regime (below $4 \mathrm{~J} / \mathrm{cm}^{2}$ ), while at the rest of the investigated metals, the two dependencies are similar.

The dependence of the darkening effect on the applied laser pulse number was also investigated. Figure 4 shows the relative reflectivity measured at $550 \mathrm{~nm}$ as a function of the number of laser pulses for the three investigated metals. The applied fluence was $9.38 \mathrm{~J} / \mathrm{cm}^{2}$ for $355 \mathrm{~nm}$ and $11.1 \mathrm{~J} / \mathrm{cm}^{2}$ for $1064 \mathrm{~nm}$. Significant decrease of the reflectivity can already be seen at the lowest (6) laser pulses in all cases. After 15 shots, only minor decrease of the normalized reflectivity values can be observed with increasing number of pulses. The measured reflectivities for all the studied metals have been dropping below 0.015 after 50 pulses.

To investigate the effect of the laser induced diffuse reflection on the measured reflectivities of the irradiated samples the spatial distribution of the scattered intensity on the surfaces was investigated at different fluences. The reflectivities were recorded using a microspectrometer with multiple objectives having different numerical apertures: $N A=0.13,0.46$, and 0.8 . Also the total reflectivity of the surface was measured using an integrating sphere $(N A=1)$. Figure 5 shows the dependence of the measured reflected intensities at $550 \mathrm{~nm}$ on the solid angle of the observation, which were calculated from the numerical aperture values mentioned previously. All the measured reflected intensities were divided by the total reflected intensities from the integrating sphere measurements in order to make the reflectivity-solid angle curves of the different surfaces easily comparable to each other. Figure 5 also contains the curves which correspond to the perfect specular and perfect diffuse surfaces, respectively. 


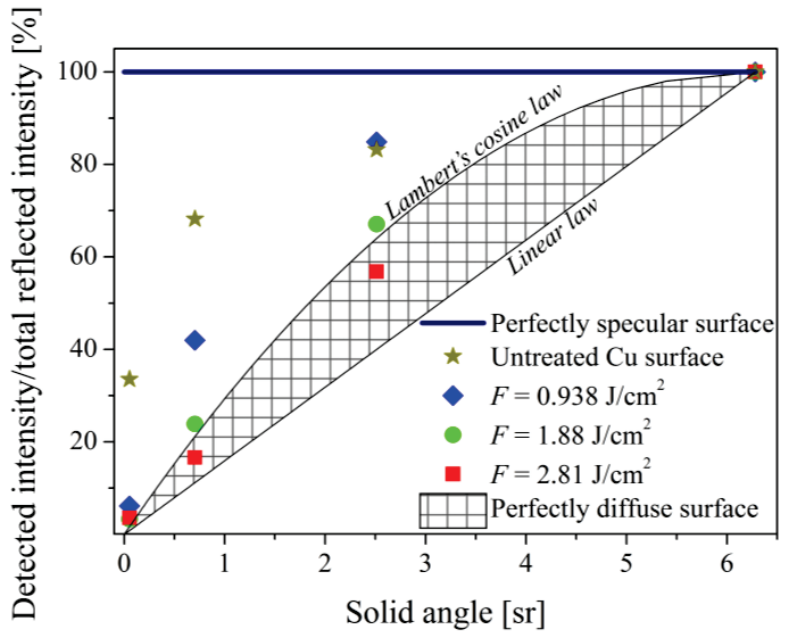

Fig. 5: The relative reflectivities of different surfaces as a function of the solid angle of observation. The investigated copper sample was irradiated with 50 laser pulses at $355 \mathrm{~nm}$ operational wavelength.

A perfect diffuse surface reflects light with isotropic luminance. When viewing a small surface element, the detected intensity would obey Lambert's cosine law, since the apparent size of the surface element is directly proportional to the cosine of the angle $\theta$ between the observer's line of sight and the surface normal. However, when considering a large diffuse surface and performing an imaging where the whole field of view is "covered" by a diffuse surface independent on the angle of sight, the size of the monitored surface will be inversely proportional to $\cos \theta$ canceling the effect of Lambert's cosine law, therefore, as a result the detected intensity will not depend on the direction of sight $(\theta)$ and therefore linear dependence is expected between the detected intensity and the solid angle.

Therefore, in real measurements, depending on the exact arrangement, the reflectivity values of a surface having a perfect diffuse reflectivity property tend between Lambert's cosine law and the previously explained linear function indicated with chequered area on Figure 5. Figure 5 reveals that even the untreated copper surface reflects some of the incident light as diffused light, while the other part is reflected in a reduced specular reflection, which behavior is due to the unpolished surface of the sample. The specular reflection further reduces when increasing the processing laser fluence. At $2.81 \mathrm{~J} / \mathrm{cm}^{2}$ and 50 pulses the measurements indicate a nearly perfect diffusely reflecting surface.

Figure 6 shows the dependence of the normalized reflectivities at $\lambda=550 \mathrm{~nm}$ on the applied laser fluence using 50 pulses measured by microspectrometer with different objectives $(N A=0.13$, 0.46 , and 0.8$)$ and integrating sphere $(N A=1)$. At low fluences (below $3 \mathrm{~J} / \mathrm{cm}^{2}$ ), the measured reflectivities are closer to each other (proportionally) than at higher fluences, which is in correspondence with the results on Figure 5, which revealed that the specular reflectivity is more dominant in the low fluence regime.

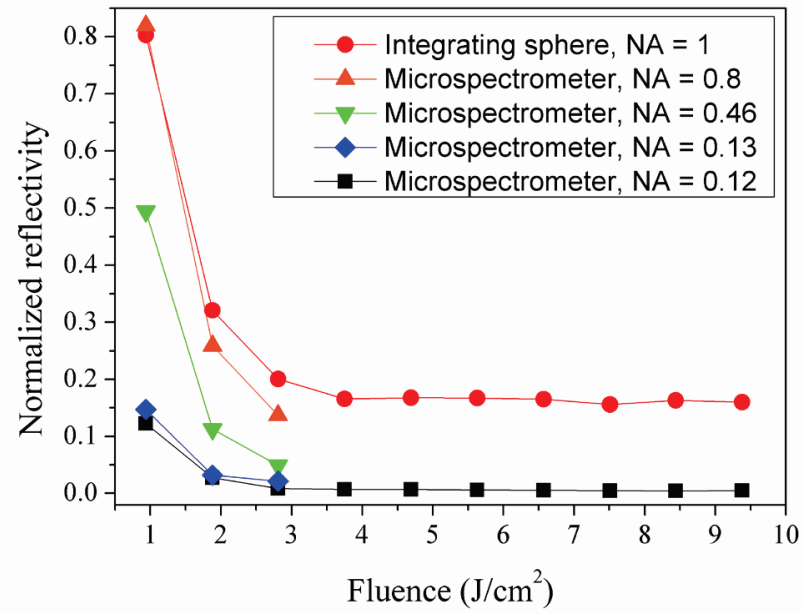

Fig. 6: Relative reflectivity measured at $550 \mathrm{~nm}$ for copper as a function of the applied laser fluence. The samples were irradiated with 50 laser pulses at $355 \mathrm{~nm}$. The measured reflectivities are normalized to the original reflectivity of the sample.

Above $3 \mathrm{~J} / \mathrm{cm}^{2}$, the surface maintains its reflectivity distribution (which is almost perfectly diffuse according to Figure 5), therefore the reflectivities measured with microspectrometer $(N A=0.12)$ and integrating sphere only differ in a constant multiplying factor in this range. Although the reflectivity values measured by integrating sphere saturate around 0.165 , it is important to note that these values are normalized to the original reflectivity of the untreated sample, of which the absolute reflectivity measured with integrating sphere and $\mathrm{BaSO}_{4}$ reference was 0.287 . Therefore, the absolute reflectivity of the dark surfaces is around 0.047 at $550 \mathrm{~nm}$.

\subsection{Morphological investigations}

To explain the results presented above, we investigated the morphology of the laser-irradiated metal samples. The developed surface structures were observed by a Hitachi S4700 scanning electron microscope (SEM) operating at $15 \mathrm{kV}$ accelerating voltage and $10 \mu \mathrm{A}$ emission current without applying any additional conductive coating on the investigated surfaces. The magnification was $50 \mathrm{k}$, at which the presence of nanometer-sized structures can be spotted.

Figure 7 shows the morphologies of the copper, gold, and silver sample surfaces after 50 pulses at different fluences and different processing wavelengths. It was found that morphology changes occur even at the lowest applied laser fluence in case of all the studied materials. More significant changes in the morphology were observed when raising the fluence, however, above $2 \mathrm{~J} / \mathrm{cm}^{2}$ fluence significant morphological differences were not observable. This shows good a correspondence with the tendencies of the measured reflectivities. It can be seen that the final structures of the illuminated gold and silver surfaces are more or less similar; however, the morphology of the treated copper sample is different. On 
the illuminated gold and silver surfaces sponge-like structures can be seen, which is formed by frozen jets and droplets having basically a few hundred nanometers size. Contrarily, in the cases of copper a more or less isomorphic basic structure is densely covered by mass of nano-aggregates (having approximately a fewnanometers linear extent), which form coral-like structures. Descended molten droplets are also noticeable on the top of the metal surfaces having various diameters from about $100 \mathrm{~nm}$ to $500 \mathrm{~nm}$. Regarding the dependence of the developed surface structures on the wavelength of the processing laser pulses; in case of copper and gold no significant difference was experienced between the studied cases, however, in case of silver the sponge-like structures on the surface are more conglutinated at the shorter processing wavelength and isolated droplets cannot be seen on them above $8 \mathrm{~J} / \mathrm{cm}^{2}$.

At fixed fluences $\left(F=11.1 \mathrm{~J} / \mathrm{cm}^{2}\right.$ for $1064 \mathrm{~nm}$ and $F=9.38 \mathrm{~J} / \mathrm{cm}^{2}$ for $\left.355 \mathrm{~nm}\right)$ we investigated the structure evolution process as a function of the pulse number (Figure 8). It was found that most of the morphological modifications of the irradiated areas took place within the first six shots for all the investigated metals. Above this value considerable structural changes cannot be noticed. These observations correspond fairly to the data of the reflectivity measurements. The wavelength of the irradiating pulses did not affect the formed morphology in case of copper and gold in the investigated range, while at silver the difference was similar as it was found in case of the fluence dependence.

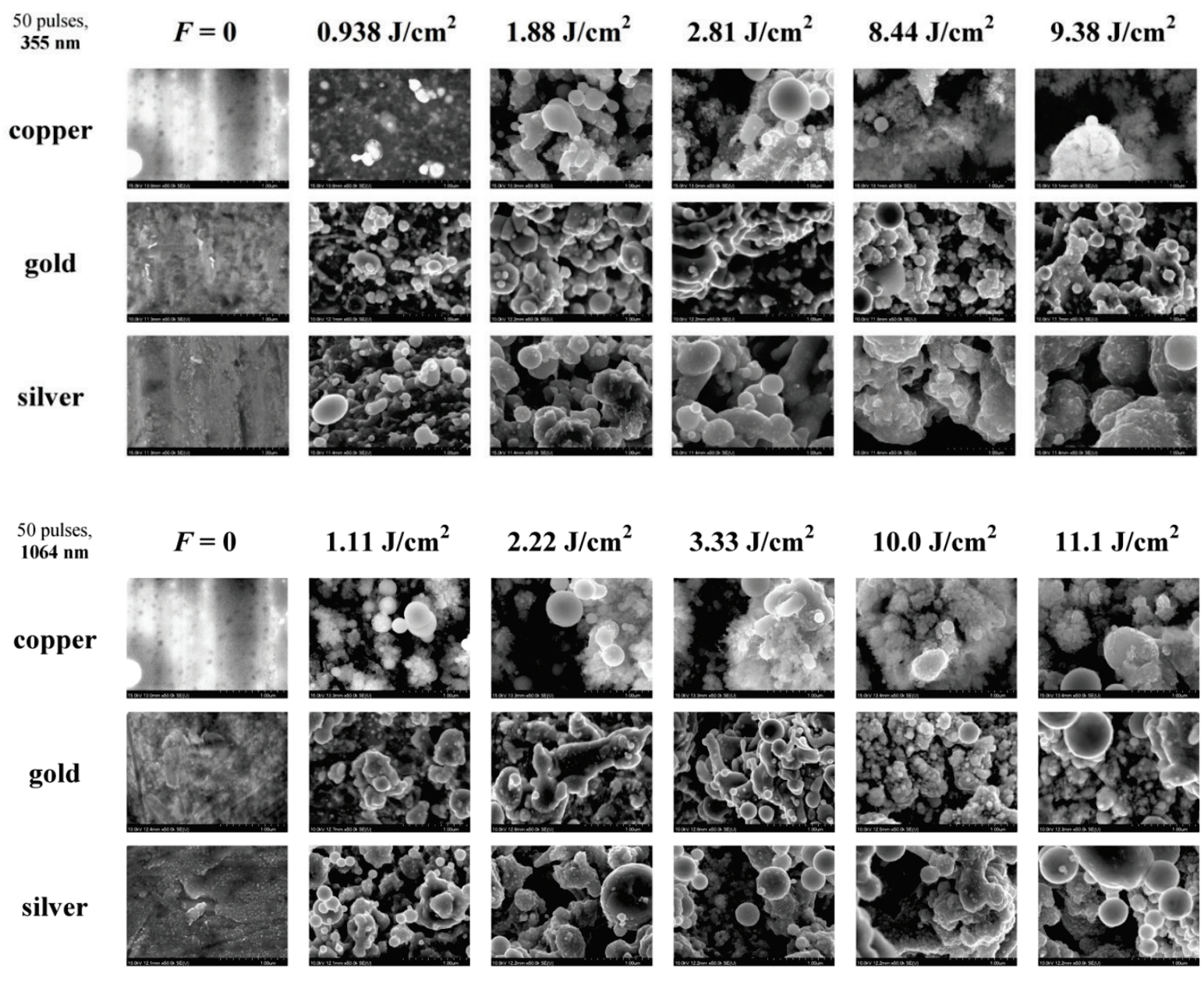

Fig. 7: High magnification $(\times 50 \mathrm{k})$ scanning electron microscopic images on the metal surfaces irradiated with different laser fluences $(F)$. The applied pulse number was 50 . The width of the images is $\sim 2.8 \mu \mathrm{m}$. 


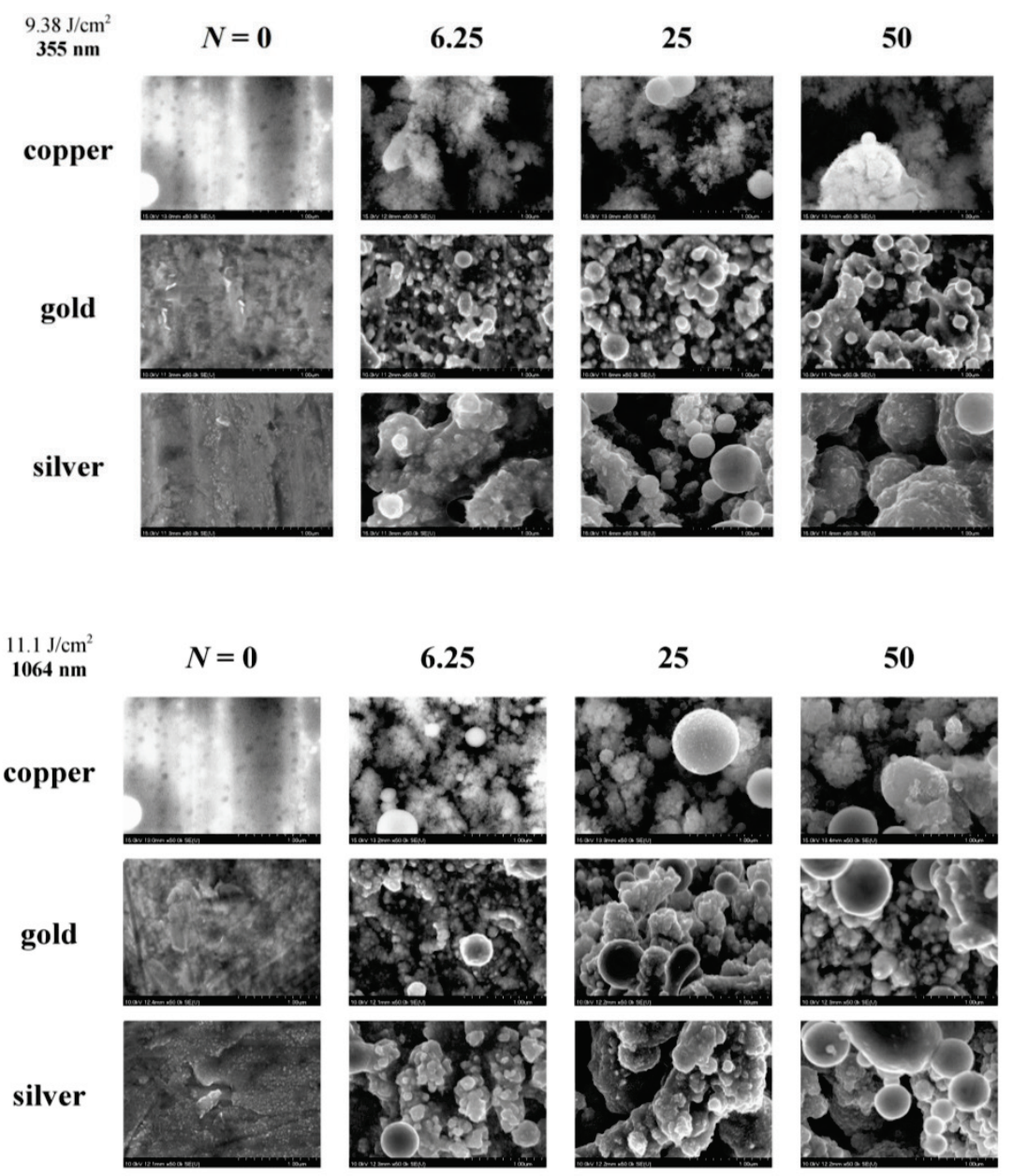

Fig. 8: High magnification $(\times 50 \mathrm{k})$ scanning electron microscopic images on the metal surfaces irradiated with different numbers of pulses $(N)$, averaged. The applied fluence was around $10 \mathrm{~J} / \mathrm{cm}^{2}$ in this case. The width of the images is $\sim 2.8 \mu \mathrm{m}$.

Figure 9 shows SEM recordings prepared on the unirradiated outer region nearby the irradiated area of the silver target (the last laser-scanned line is shown on the upper part of the left image by a dashed line). The figure demonstrates that the ablated material redeposits not only within the irradiated area, but can travel far away from it, too. Considerable amounts of nanoparticles and molten and resolidified nano- and microdroplets were found even at a distance of $200 \mu \mathrm{m}$ from the last scanned line, which are revealed on high $(\times 50 \mathrm{k})$ magnification images at the right side of the figure.

Our observations imply that the deposition of surface nano- and microstructures is a key element in the darkening of the investigated metals. SEM images suggest that formation of these structures is foregone by the melting (or even boiling) and refreezing processes due to the high temperature reached by the absorption of the applied laser energy. To get deeper understanding to this process, the spatial and temporal temperature distributions in picosecond laser-irradiated metals were calculated at different fluences $\left(1.11-11.1 \mathrm{~J} / \mathrm{cm}^{2}\right)$ according to the experimental data. In this thermal model the one-dimensional heat transfer equation was solved by the finite element method. The absorbed laser energy in the irradiated metals was considered to be a heat source, which has a Gaussian temporal profile and an exponential spatial (depth) profile according to the Beer-Lambert absorption law. The wavelength of the irradiating laser pulse $(\lambda=1064 \mathrm{~nm})$ was taken into account in an implicit manner, where the values of wavelength-dependent material parameters (absorption coefficient and initial reflectivity) were given at the chosen wavelength. The relevant thermodynamic parameters of the metals (specific heat capacity and thermal conductivity) were included as a function of the temperature and latent heats were also taken into account, thus phase changes could be simulated during the thermal heating process. It was found that the surface temperature of the irradiated metals exceeds their melting and boiling temperature already at the lowest applied fluence $\left(1.11 \mathrm{~J} / \mathrm{cm}^{2}\right)$. This calculated result is in agreement with the SEM images, where masses of molten and resolidified droplets can be seen. Our calculations were limited to the first incident laser pulse, since the forthcoming pulses reaches a surface with modified morphological, reflective and thermal properties, which extremely encumber the simulation of the whole micro- and nanomachining process. 


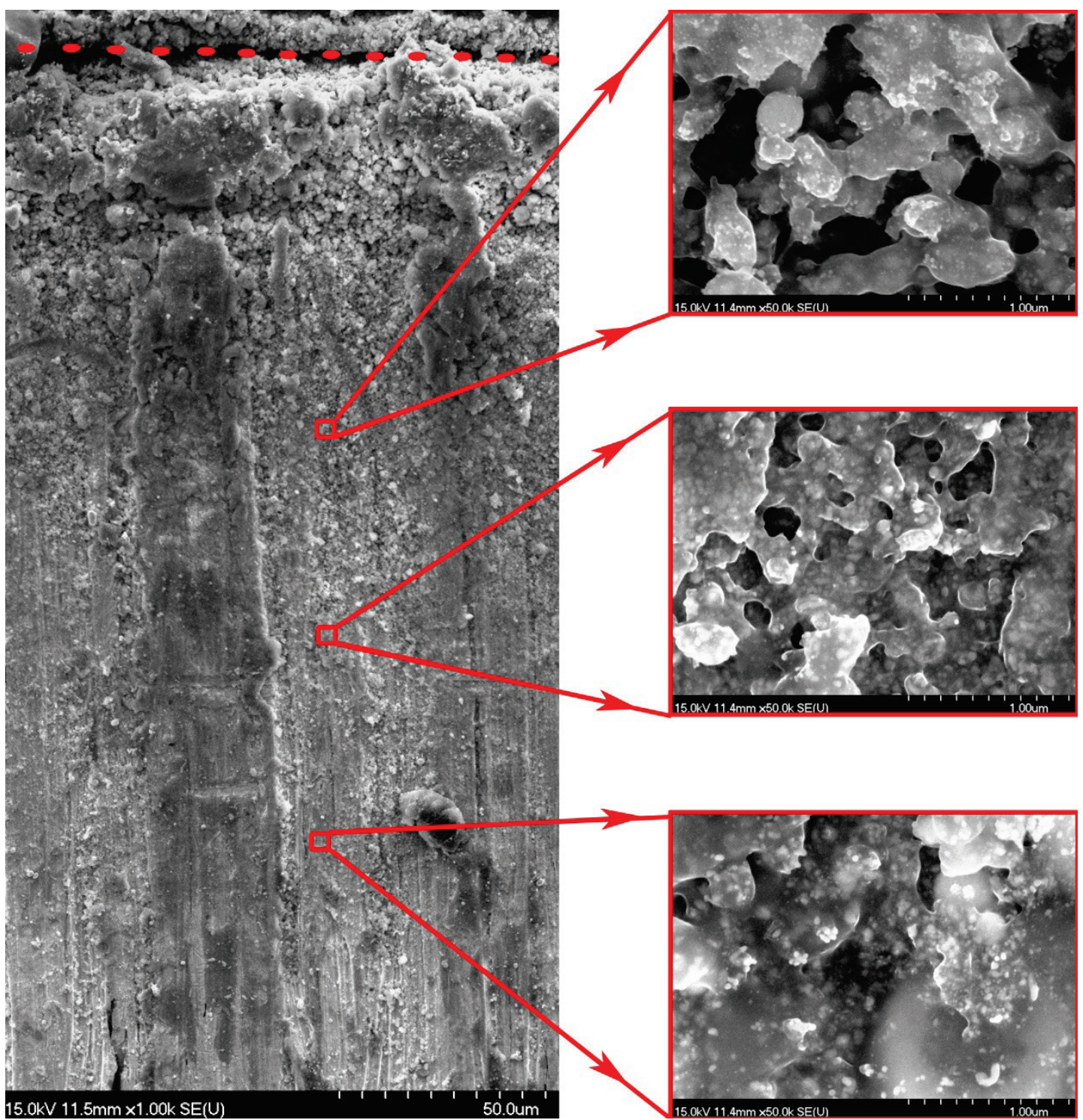

Fig. 9: Scanning electron microscopic images of the unirradiated marginal region of the treated silver sample, where maximum fluence $\left(F=9.38 \mathrm{~mJ} / \mathrm{cm}^{2}\right)$ and pulse number $(N=50)$ were applied at $355 \mathrm{~nm}$ irradiating wavelength. The width of the image on the left is $\sim 140 \mu \mathrm{m}(\times 1 \mathrm{k}$ magnification $)$; while on the right the high magnification $(\times 50 \mathrm{k})$ pictures have $\sim 2.8 \mu \mathrm{m}$ width.

The formation of filament-like structures on the irradiated copper surface can be due to the interaction of the ablation plume with the high-pressure (1 bar) ambient gas, which results in particle condensation and cluster formation near the irradiated terrain in the early ablation stage, when the ablated plume is still expanding rapidly and its density is high enough [23]. For the same reason picosecond ablation is suitable for generation of nanoparticles $[24,25]$. The ablated nanoparticles collide with each other and the ambient gas molecules, which causes a certain percentage of ejected particles to reflect backwards onto the target surface [26]. Morozov proved by Monte Carlo simulations that up to $20 \%$ of the atoms could be reflected towards the direction of the irradiated surface depending on the background pressure [27]. It has also been demonstrated by Égerházi et al. that this backward scattering of the ablated fragments has practical application possibilities for depositing thinlayers [28]. Rode et al. described that a diffusion limited aggregation can form coral-like structures of united clusters during ablation of glassy carbon with a high repetition rate femtosecond laser [23]. In our previous experiments [10] similar copper, silver, and gold bulk samples were irradiated by femtosecond Ti:sapphire laser at different fluence and pulse number values. In the femtosecond case the filament-like structures have more significant dominance on the surface (especially on copper and gold) than it was observed in the picosecond experiments (Figure 10). It has to be noted that in Figure 10 the applied pulse number was 50 in the picosecond case and was 100 during the femtosecond irradiations, however the morphology of the irradiated area only changes slightly between 50 and 100 pulses because of the observed saturation in the femtosecond experiments 
at $2000 \mathrm{~mJ} / \mathrm{cm}^{2}$ [10], ], which allows comparing the two results in a reasonable way. The explanation of the observed behaviour is that femtosecond irradiation results in a more intensive evaporation of the irradiated material because of the higher power density, which leads to the increase of the plasma density. Due to this more clusters were being formed during the diffusion-limited aggregation process.

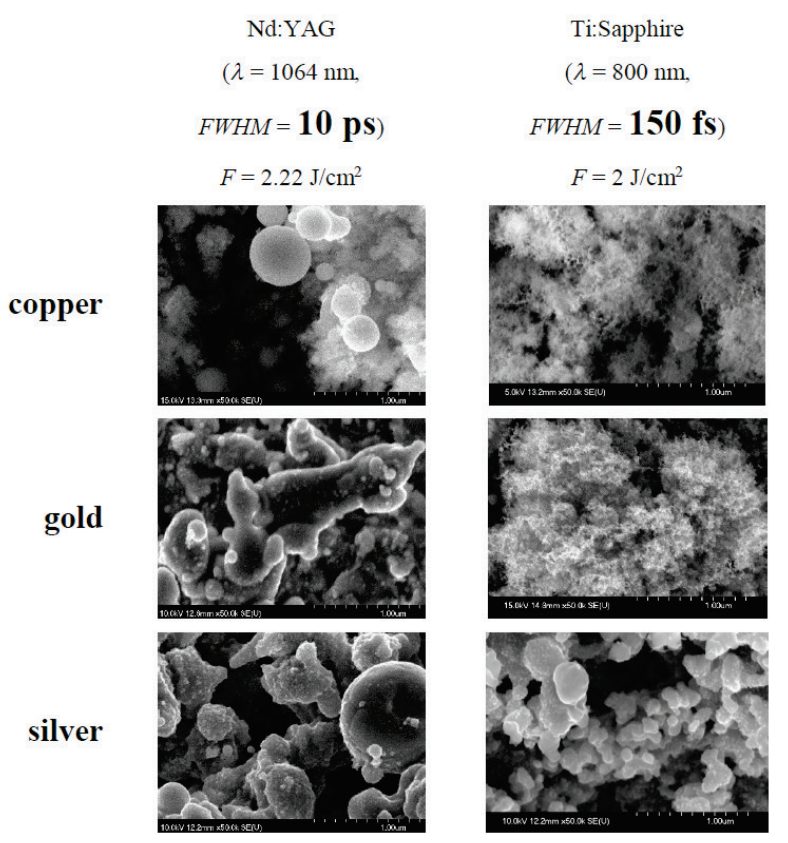

Fig. 10: SEM images of the irradiated copper, gold and silver surfaces for two different pulse durations, $10 \mathrm{ps}$ (left), and 150 fs (right). The applied pulse number was 50 in the picosecond case and 100 during the femtosecond experiment. The width of the images is $\sim 2.8 \mu \mathrm{m}$.

The samples were also investigated by X-ray photoelectron spectroscopy to reveal the possible role of laser-induced chemical modifications in the reflectivity decrease mechanism. The XPS measurements showed the presence of copper(II) oxide on the surface of the irradiated copper samples, while in case of silver and gold changings in the chemical composition cannot be proved. Detailed study on surface composition and structure with quantitative X-ray diffraction (XRD) analysis was performed by Fan et al., where they found signs of oxidation on copper targets irradiated by $10 \mathrm{ps}$ YVO4 laser system at a central wavelength of $1064 \mathrm{~nm}$. However, they speculated that the composition change of the sample surface was not the main reason for the dramatically enhanced absorption [11]. Moreover, it has been shown by Tang et al. in the process of nanosecond laser blackening of copper that the optical contribution of the oxide layer is on average approximately $3 \%$ in the visible range and increases to $10 \%$ on average throughout the infrared spectral range [29].

\section{Conclusions}

Our experiments have shown that picosecond $\mathrm{Nd}$ YYA laser irradiation under the presented experimental conditions is an appropriate method to prepare non-reflective surface structures on copper, gold, and silver bulk materials using UV $(355 \mathrm{~nm})$ or IR $(1064 \mathrm{~nm})$ wavelengths. It was found that the normalized reflectivity of the laser-treated areas drastically diminishes in a broad spectral range with increasing fluence reaching lower than $3 \%$ in all cases above $3 \mathrm{~J} / \mathrm{cm}^{2}$ laser fluence. The dependence of the darkening of the treated spots on the applied laser pulse number was also investigated and it was found that the most significant decrease in the measured reflectivity occurs within the first six pulses. After 15 shots, only minor further decrease was observed. The light scattering properties of the areas irradiated at different fluences were investigated and it was found that due to the laser irradiation the initially specular reflectivity of the surface was reduced with the increasing laser fluence and above $3 \mathrm{~J} / \mathrm{cm}^{2}$ a nearly ideal diffusely scattering surface with low total reflectivity was produced. The scanning electron microscopic investigations showed that two types of nanostructures can be responsible for the reflectivity decrease depending on the type of the illuminated metal. On the silver and gold samples sponge-like structures characterize the surface, which are formed by frozen jets and droplets with hundreds of nanometers diameter. In case of copper an additional structure type was found, which consists of nanofibre mass having few nanometers size on a basic structure, which is more or less similar to the other two metals' surfaces. In the development of the sponge-like pattern the ejection and falling back of molten metal droplets and jets play an important role, while the forming of the observed foam-like structure in case of copper can be due to the backscattering and aggregation of nanoparticles with few nanometers dimensions.

We believe that such dark metals can be used as wavelength-independent anti-reflective materials, which are darkened in their substance for applications where high efficiency and wide wavelength range radiation capturing are as important as high conductivity properties: like photovoltaic elements, light meters, laser surgery etc. The main advantage of this technique is that it does not require the application of special coatings prepared by materials which are different from the substrate material; therefore, the thermal, electronic etc. matching of these is not necessary.

\section{Acknowledgements}

This research was supported by the European Union and the State of Hungary, co-financed by the European Social Fund through projects 'Biological and environmental responses initiated by new functional materials' (TÁMOP-4.2.2.A-11/1/KONV-2012-0047), „Supercomputer, the national virtual lab" (TÁMOP4.2.2.C-11/1/KONV-2012-0010), the DAAD researcher exchange program (no. 56266271) and in the framework of „National Excellence Program - Elaborating and operating an inland student and researcher personal 
support system convergence program" (TÁMOP 4.2.4. A/2-11-1-2012-0001).

\section{References}

[1] A.Y. Vorobyev and C. Guo: Laser \& Photonics Rev., 7, (2013) 385.

[2] A.Y. Vorobyev and C. Guo: Adv. Mech. Eng. 2010, (2010) 452749.

[3] A.Y. Vorobyev and C. Guo: Optics Express, 14, (2006) 2164.

[4] Md. S. Ahsan, F. Ahmed, Y. G. Kim, M. S. Lee and M. B.G. Jun: App. Surf. Sci., 257, (2011) 7771.

[5] A.Y. Vorobyev and C. Guo: Opt. Photonics News, 19, (2008) 30.

[6] A.Y. Vorobyev and C. Guo: Appl. Phys. Lett., 92, (2007) 041914.

[7] A.Y. Vorobyev and C. Guo: Phys. Rev. B, 72, (2005) 195422.

[8] Y. Vorobyev and C. Guo: J. Appl. Phys., 104, (2008) 053516.

[9] T.-F. Yao, P.-H. Wu, T.-M. Wu, C.-W. Cheng and S.-Y. Yang: Microelectron. Eng., 88, (2011) 2908.

[10]B. Hopp, T. Smausz, T. Csizmadia, Cs. Vass, Cs. Tápai, B. Kiss, M. Ehrhardt, P. Lorenz and K. Zimmer: Appl. Phys. A, 113 (2013) 291.

[11]P. Fan, M. Zhong, L. Li, T. Huang and H. Zhang: Opt. Express, 21, (2013) 11628.

[12] Y.C. Guan, W. Zhou, H. Y. Zheng and Z.L. Li: App. Surf. Sci., 280, (2013) 462.

[13] Y.C. Guan, W. Zhou, Z.L. Li and H. Y. Zheng: Optics and Lasers in Engineering, 52, (2014) 35.

[14]A.Y. Vorobyev and C. Guo: Opt. Express, 19, (2011) A1031.
[15]A.Y. Vorobyev and C. Guo: Appl. Phys. A, 86, (2007) 321.

[16] B. K. Nayak and M. C. Gupta: Optics and Lasers in Engineering, 48, (2010) 940.

[17] R. Torres, V. Vervisch, M. Halbwax, T. Sarnet, P. Delaporte, M. Sentis, J. Ferreira, D. Barakel, S. Bastide, F. Torregrosa, H. Etienne and L. Roux: J. Optoelectron, Adv. Mater., 12, (2010) 621.

[18] V. V. Iyengar, B. K. Nayak, K. L. More, H. M. Meyer, M. D. Biegalski, J. V. Li and M. C. Gupta: Sol. Energy Mat. \& Sol. Cells, 95, (2011) 2745.

[19] M. Richard, F. Richard, A. Karger, J. Carey and E. Mazur: Appl. Opt., 45, (2006) 8825.

[20] A.Y. Vorobyev and C. Guo: J. Appl. Phys., 110, (2011) 043102.

[21] A.Y. Vorobyev and C. Guo: Appl. Phys. A, 86, (2007) 235.

[22] K.-E. Peiponent and T. Tsuboi: Opt. Laser Technol., 22, (1990) 127.

[23] A.V. Rode, E.G. Gamaly and B. Luther-Davies: Appl. Phys. A, 70, (2000) 135.

[24]A. Schwenke, P. Wagener, S. Nolte and S. Barcikowski: Appl. Phys. A, 104, (2011) 77.

[25] M. Guillermin, J. P. Colombier, S. Valette, E. Audouard, F. Garrelie and R. Stoian: Phys. Rev. B, $82,(2010) 035430$.

[26] M. Biscan and S. Milosevic: Spectrochim. Acta B 68, (2012) 34.

[27] A.A. Morozov: Appl. Phys. A, 79, (2004) 997.

[28]L. Égerházi, Zs. Geretovszky and T. Szörényi: Appl. Surf. Sci., 247, (2005) 182.

[29] G. Tang, A. C. Hourd and A. Abdolvand: Appl. Phys. Lett., 101, (2012) 231902.

(Received: June 15, 2014, Accepted: January 22, 2015) 